\title{
Diversities in Approach to End-of-Life: $A$ view from Britain of the qualitative literature
}

\author{
Kip Jones, $\mathrm{PhD}$ \\ ESRC RESEARCH FELLOW
}

CENTRE FOR EVIDENCE IN ETHNICITY, HEALTH \& DiVERSITY

MaRY SEACOLE ReSEARCH CENTRE

DE MONTFORT UNIVERSITY 


\section{Executive Summary}

The process of dying, perhaps more than any other moment in the course of medical care, can accentuate cultural difference between patients, families, and providers" (Krakauer, Crenner \& Fox 2002: 184).

Brief: to investigate qualitative literature on end-of-life and ethnicity/race/diversity and conduct the review systematically, using qualitative methods and philosophical concepts.

Method: An enlightened attitude is employed that approaches diversities in practice, consolidated from a world-view of qualitative evidence, as new information beneficial to the wider society as a whole-expanding policy, practice and the collective conscience in the UK and elsewhere.

Outcome: The usefulness of the evidence to research, policy and service-user circles contributes to the larger society's dialogue on death and dying more generally, encouraging participation in the common habitus through discovery of individual and group differences.

\section{Key findings include:}

- The review of qualitative literature on palliative care is minimal, with little mention of ethnicity/race/diversity; palliative care generally pays little attention to qualitative methods.

- As the UK becomes more culturally diverse, the risk to minorities of poor end-of-life care due to cultural misunderstandings is likely to grow just as it has in the US.

- The rights of families to medical knowledge and their roles in decisionmaking are just as valid, inalienable and crucial to the cultural belief systems of many ethic minority communities as are Western patient autonomy models.

- Collective research findings consistently suggest that choices involving endof-life medical treatment decisions may be more related to ethnicity and culture than to age, education, socio-economic status, or other variables.

- Healthcare providers must recognise that the basic values, principles and assumptions of western medicine and bioethics are themselves historically situated and culturally determined. 
- The 'cookbook' approach to diversity creates new myths or stereotypes, and compounds this with inaccuracies or misunderstandings. Aspects other than formal religious beliefs are overlooked and not all members of an ethnic group will routinely follow the beliefs of a specific faith.

- There is a wide variation of beliefs and behaviours within any ethnic population.

- Building blocks to understanding include time effects such as age and the life course, cohort effects and generational differences, and historical trends and period effects.

- The hospice movement has as its goal the facilitation of mental and spiritual preparation for death, including the meaning-making aspects of hospice care and a holistic interpretation of spiritual care.

\section{Ethical Considerations in End-of-Life decision-making:}

$\diamond$ End-of-life decision-making is often couched in religious contexts.

$\diamond$ Mistrust of human medical technology is often in play.

$\Delta$ There is often a giving up of control to family members by patients.

$\Delta$ Ethical questions of disclosure and non-disclosure are compounded by considerations of culture and faith.

$\diamond$ A model of the self is embedded within a network of social relations.

$\diamond$ The reality of cultural pluralism must be realised in policy.

$\Delta$ Reconciling the goal of equality with the reality of difference is crucial.

$\Delta$ 'What is the family's place in ethical discussions of end-of-life decisionmaking?' enters into any discussion on ethics and end-of-life care.

Much has been learned from Aids service provision about people's ability to meet the challenges of care, particularly in the face of negativity and prejudice and ways to develop new practice through synthesis and integration of older models.

Family involvement in end-of-life decision-making cannot be ignored: persons from a wide range of ethnic and/or racial backgrounds (including the white population) prefer family involvement.

A common theme throughout the studies reviewed is a need for a sensitivity to the varying expectations and mix of involvement of patients, practioners and family (including concepts of extended family and significant others) in end-of-life care and the need for information-sharing and decision-making amongst these parties. 


\title{
Diversities in Approach to End-of-Life: \\ $A$ view from Britain of the qualitative literature
}

\author{
Kip Jones, PhD \\ ESRC RESEARCH FELLOW \\ Centre for Evidence in Ethnicity, Health \& Diversity \\ Mary Seacole Research Centre \\ DE MONTFORT UNIVERSITY
}

\begin{abstract}
Overview
This paper is a consideration of diversities in approach to a time of life that all of us face eventually: the end of life or death. How that process (journey) is conceptualised and contextualised by individuals from diverse backgrounds (ethnic/racial and so forth) is at the foundation of this review. When death approaches, "even patients and families who do not usually follow traditional practices may resort to them. The process of dying, perhaps more than any other moment in the course of medical care, can accentuate cultural difference between patients, families, and providers" (Krakauer, Crenner \& Fox 2002: 184). Rather than simply a study of esoteric or exotic practices carried out by groups that might be portrayed as 'other', the subject is of particular interest because dying is a universal process. By such an approach, the aim is to move policy and practice in the field (in the UK and elsewhere) beyond the measures that have been trotted out over the past 20 years: diversity training and ethnic headcounts (Kundnani 2002: 6).
\end{abstract}

Because some might approach dying and death from a different viewpoint, culture or context, the study of alternative approaches to end-of-life has the potential to contribute to the larger society's dialogue on death and dying more generally. It is in this spirit of participating in the common habitus through discovery of individual and group differences that this review is carried out. The paper investigates qualitative literature on end-of-life and ethnicity/race/diversity and was conducted using qualitative methods and philosophical concepts (see Jones 2003 for an elaboration).

\section{Mapping the exercise}

The approach that was taken in this review of the qualitative literature consolidates a world-view of the evidence on end-of-life and ethnicity/race/diversity, and is a tangential and animated one-at times amorphous and uncrystallized. Qualitative work is in constant, dynamic flux, but moving toward some end-point in an evolutionary way. There are efforts by the mind to concretise meaning and the qualitative dimension has an integrative function for the researcher and so it was with this review process. Qualitative research often begins in an unstructured way without a question, but looks for questions throughout the process.

In this qualitative review, databases, expanding and contracting like an accordion's bellows, were compiled using the usual search tools. At each step along the way, concepts as well as queries began to emerge. Pirolli and Card's information foraging theory (IFT) (Pirolli \& Card 1995) comes into play here. The theory states that people will behave in bounded rational ways to optimise their interactions with 
technology in order to achieve their goals and interests (Pirolli \& Card 1998: 1). The authors explain Foraging Theory and Information Patch models:

Information relevant to a user's information needs may reside in piles of documents, file drawers, office book shelves, libraries, or in various online collections. Often the user has to navigate from one information patch to another, perhaps from one pile to another, or from one on-line collection to another. Often the user is faced with a decision much like our imaginary bird: is it better to continue foraging through the current patch or is it better to seek out another patch? Information Patch models provide an analysis of this problem. (1998: 2).

In their study of the Scatter/Gatherer system, the authors show how searches using internet-based tools are influenced by personal choices and decision-making, including the building in of 'costs' of the information retrieval itself. Gestalt principles of cognitive psychology also come into focus in how people perceive patterns in the retrieval process. IFT supports claims for a tangential, intuitive and personal approach to systematic review searches, making often-stated declarations of the abilities of systematic review searches to be replicated questionable.

Qualitative principles were employed throughout this review's process; for example, the use of the qualitative method of 'saturation' made sense for the literature searches in this study. The logic of qualitative sampling rests not so much on generalisability, nor on representativeness, but on notions of saturation, that is, the point at which no new insights are likely to be obtained. Data saturation occurs in qualitative research when the researcher is no longer hearing or seeing new information. For instance, when one paper after another reported on a particular ethnic group's approach to end-of-life decision-making, it became clear that the topic had been covered or saturated. Another example of where saturation was reached was when 'cookbook' approaches to particular faiths and their 'peculiarities' in the face of death became redundant. The originators of the metaethnographic approach to systematic review mirrored in this review's effort, Noblit and Hare (Noblit \& Hare 1988), caution: "Unless there is some substantive reason for an exhaustive search, generalizing from all studies of a particular setting yields trite conclusions" (1988: 28).

The qualitative technique of 'snowballing' also came into play in literature searches. Snowballing traditionally refers to one informant suggesting that the researcher talk to another potential informant. In this literature search, the principle was used when a paper led to other sources-for instance, through citations listed in the bibliography, in conducting follow-up searches using the author's name to find more of her/his work, by tracking down electronic leads published in the papers or contacting researchers personally. Working in this tangential way, the review takes on the hallmarks of a polyvocal dialogue.

The following databases were used to conduct the searches:

PubMed Medline at the National Library of Medicine; [Zetoc access to British Library's Electronic Table of Contents (ETOC) through MIMAS (Manchester Information and Associated Services)]; ASSIA (Applied Social Sciences Index and Abstracts at Cambridge Scientific Abstracts), British Library 
and British Library's Inside Web; SIGLE (System for Information on Grey Literature in Europe); CINAHL (database for nursing and allied health literature); PsychInfo; International Bibliography of the Social Sciences; and the gateways: SOSIG (Social Science Information Gateway) and Science Direct; web searches were carried out using Google.

The search terms included the following in a variety of configurations: end-of-life, palliative, 'palliative care', 'death and dying'; ethnic, ethnicity, race, diversity, culture, and multi-cultural; all were combined with the term 'qualitative'. A general database of papers on end-of-life and race/ethnicity/diversity was compiled, consisting of 227 papers. The final database of qualitative papers is comprised of 119 references, although this has been fluid throughout the process, with numbers rising and falling, then rising again as the review crystallized both questions and concepts.

The reviewer read all of the available papers in the compiled qualitative database in full. A method of 'system and noise', developed by Higginson, Goodwin, Edwards and Norman (2002) and elaborated by this researcher (see Jones 2003b), was used to select papers for inclusion in this report. As reading produced a growing sense of both the context and shape of the existing literature on end-of-life and race/ethnicity/diversity, key questions developed for further analysis later, with the aim of using the narrative method of analysis and consensus suggested in a previous paper (Jones 2003b). What is reported here is the groundwork for the use of such a qualitative analysis protocol.

\section{Codicil}

To claim that this review's literature search is final, complete or the absolute last word in the area under investigation would be an unproductive conceit. Pragmatic concerns, such as funds available (see Pirolli \& Card 1998) to pay for commercial database searches, time available to the investigator and deadlines for projects due, profoundly affect the search strategies and the results of the best of intensions of systematic reviewers. Secondly, any literature search is done in a certain and specific time/space yet must strive to achieve some semblance of the shifting contexts and the fleeting nature of human reality (Harré 1997: 278). The same search, done on a different day, may make subtle or not so subtle differences in the results retrieved (this is particularly evident in the results of web searches, for example).

What is required of systematic reviews are a consideration of the usefulness of the evidence to the areas under review and how any compiled information may (or may not) be helpful in research, policy and service-user circles. What is also needed is less emphasis on the 'rigour' of the intricacies and convolutions of the search procedure itself; to put it simply, less fussing over the preparation and more attention to the meal. Perhaps, in the end, paying someone to do the literature searches (for example, search experts at the British Library) might make better use of funds available and free up project research staff for the reviewing, analysing and synthesising work at hand in evidence reviews. These are the tasks so necessary in order to produce documents of benefit to policy makers, service users and their representatives. 


\section{A discussion of terms and terminology}

By attempting to synthesise documents where the results have the potential to develop models that are more than the sum of their parts, a process of integration is necessary. Gergen alerts us to the concept that this is often accomplished by the integration of preceding intelligibilities and realignment of existing ones and their practices (Gergen 2001: 430). This process is accomplished linguistically, that is, with language, and constructed socially to make its case. A mixture of researchers and policy makers that comprise any field develop precise terminology to describe and support their areas of interest. The very words they use "derive their meaning from the attempt of people to coordinate their actions within various communities" (Gergen 1997: 6). Their particular language evolves into symbols over time with the ability to 'telegraph,' in a concise way, assumed knowledge and background information to their audiences. These assorted research, policy and interest-group communities subsequently produce papers and reports comprised of a kind of 'shop talk' that heightens participation in the language game, enabling them to ring-fence their areas of expertise. This dialogue results in producing icons with the accumulated power to persuade, to convince, to establish authority and to represent authenticity, but often skewing and/or stifling wider community discourse and input through the process itself.

Within the literature on ethnicity/race/diversity, for example, this evolutionary process becomes particularly heightened. The words chosen, which are appropriate (politically correct) to use in discussions on issues of ethnicity, race and/or diversity, vary and change over time and from group to group. In reviewing the literature on end-of-life, for example, searches using the root term 'ethnic' turn up literature from many British sources. Using the search term 'race' brings forth mostly American studies. It would be simplistic to come away from this finding assuming that nationalistic differences (historic, political and/or linguistic) alone produce this anomaly. The British literature seems particularly to back away from the term 'race' and yet, the term 'racism' is quite prevalent in UK policy literature.

Why is there this apparent reluctance to use the root word, 'race'? There are attempts to discount the use of the term 'race', particularly in the British literature. Defining 'ethnicity' in the US, Nickerson (2001) remarks, "Although not commonly acknowledged, many people look first toward visible physical characteristics and/or key behaviors (e.g., language) when categorizing individuals by ethnicity" (Nickerson 2001: 2). By Nickerson's own definition, 'race' "clearly represents a socio/political/historical construct used as a classification system based on patterns of visible physical characteristics" (2001: 2). According to Nickerson's definitions, then, race and ethnicity can be interchangeable. Several scholars, however, note the increasing tendency to substitute ethnicity for race. Kenyatta and Tai (1997) conclude that some researchers "use ethnicity interchangeably with race because they are still uncomfortable with race, racism and its role" (Kenyatta \& Tai [1997], cited in NCDDR 2002: 3). In abandoning the concept of race, there is a serious tendency to abandon discussions of power, domination, and group conflict. In works on ethnicity, the discussions quickly turn to matters of culture and identity rather than issues of political power or powerlessness (Kenyatta \& Tai [1997], cited in NCDDR 2002: 3 ). 
Is something else more obtuse, then, being reflected just beneath the surface of language use in the British literature? Is a softening of the language used, perhaps, to make the terms more palatable? (The sound of the multi-syllabic word, 'ethnicity', is more quieting than the brash word, 'race'.) Is there something about white British culture and its tendency to be nonconfrontational, perhaps by averting its gaze from what it perceives might be unpleasant? Is it plausible that some, by not using the term 'race', believe that racism will disappear? US historian Manning Marable warns: "The UK ignores at its peril the subject of race" (BBC-TV FOUR 2003).

Or does this elaborate language game represent a 'circling of the linguistic wagons' by research and policy interest groups to protect their patch, thus avoiding reflecting common usage as well as common sense? Too often this kind of needless refinement (or should we say neutering?) of language produces terms that make their way from the research and policy literature into popular culture, thus neutralising common concerns amongst the public and, by the process, politicising research. The dangers of ethnocentricity and naïve promotion of multiculturalism lie in the fact the fight against racism may very well be transformed into a fight for culture (Kundnami 2002: 5). Researchers must be constantly conscious of the contribution that they may or may not make to the politics of race through language. If, as researchers, systematic reviewers or policy makers, we are to participate in "enriching the forms of societal practice", as Gergen (2001: 431) has invoked us to do, then perhaps it is time to be more reflective of the populations we study and more directly echo their 'voice'-albeit, at times, through the forceful and unsettling polyvocal language of the quotidian. This is best accomplished by qualitative methods. In pursuing this goal, researchers need to be alerted to the fact that the concepts of race, ethnicity and culture are sometimes used to describe the same thing (NCDDR 2002: 2), at least in the wider arena of society at-large

These critical issues of terminology extend to the literature on end-of-life as well. Werth, Blevins, Toussaint and Durham (2002) caution: "In writing about cultural diversity, summarizing research on various groups and using case examples, both authors and readers alike run the risk of stereotyping people" (2002: 205). Using the term 'cultural diversity', the authors speak of 'culture' as referring to more than merely ethnicity. They cite Krakauer, Crenner and Fox's definition of culture as "a constellation of shared meanings, values, rituals and modes of interacting with others that determines how people view and make sense of the world" (Kakauer et al 2002 cited in Werth et al 2002: 204). Krakauer et al elaborate: "Although the definitions of 'ethnicity' and 'culture' overlap, 'ethnicity' denotes, at least in part, a shared genetic or geographical origin" (Krakauer et al 2002: 184). Following a view that diversity encompasses respect for the unique needs and wishes of each patient's end-of-life wishes, Werth et al make the case that individuals in a group run the range of the values, attitudes and actions of the group itself (Werth et al 2002: 205). The concept of dualities of identity, particularly amongst minority community members, also comes into play: how a person identifies her/himself within her/himself can often be at odds with how she/he identifies her/himself in respect to the identity constructed by, and/or for, the larger society. In addition, the varieties and complexities of ethnic identities become even less easy to categorise and define when speaking of second, third or more generations of immigrant offspring. 
Although vast cultural differences exist in models of 'the individual', (more will be said later about cultural differences in concepts of individual 'rights' in terms of palliative care and end-of-life choices and individuality as a cultural concept in itself vis à vis a range of cultural constructs of the individual), it is important to note that individual differences-no matter what the culture-cannot be reduced to concepts of race, gender and/or social class, nor can they be simplified as variations around these 'norms' (Meachum 1999: 141). In fact, the concept of the 'norm' is as outmoded as it is unhelpful, at least in post-modern times; in considerations of ethnicity/race/diversity, its use is more than suspect (see Calasanti 1996: 148). The concept of the ethnic group classification (see White 2002: 1) in research is ultimately a statistician's slight-of-hand, at times inadvertently promulgating historical racism or replicating current discomfort around issues of race. For example, the 2001 UK census, for the first time, counts the religious affiliations of the population, "a move widely thought to reflect the need to measure the size of the Muslim population" (Kundnani 2002: 8).

The term 'palliative' can also be problematic. Payne, Sheldon, Jarrett, Large, Smith, Davis, Turner and George (2000) explain:

In Britain, terminology relating to end-of-life care has undergone a number of transitions, from hospice care and terminal care in the earl phases of the hospice movement to palliative care towards the end of the last century. More recently, supportive care has emerged as a popular term with the context of noncurative treatments provided to cancer patients. While the term end-of-life care was first applied to care of dying patients in Canada, this term is now widely used in North America. ... The early hospice movement was unambiguously concerned with terminal care predominantly for those with cancer; ...the transition in terminology from "terminal care" to "palliative care" reflects a "death denying" tendency (2000: 396).

According to Foucault, discourses do not just reflect or represent social entities and relations, they construct them. It is argued that the changing terminology of palliative care reflects emerging and competing ways to talk about the care provided to dying patients. It is a social and political process, which displays broader tensions and reveals power positions in society (2000: 400).

\section{Reviewing the reviews}

"Although major efforts have been undertaken in the past decade to improve endof-life care, there is growing evidence that improvements are not reaching those at especially high risk for inadequate end-of life care: minority patients" (Krakauer et al 2002: 182). Before 1997, according to Knebel (2002: 1), end-of-life research received little attention, even in the US. Since 1997, there has been an escalation in the number and breadth of end-of-life studies funded by various US government agencies and focus is expanding to include topics such as cultural and ethnic influences in end-of-life care (2002: 1). In reviewing the research initiatives to improve end-of-life care, Knebel uncovers current efforts in the areas of research, education and community involvement. 
The review of qualitative literature on palliative care itself is minimal and what exists has been carried out in nursing studies, for the most part. One recent review, that purports to be 'A Qualitative Systematic Literature Review of Patient Preferences' (Higginson \& Sen-Gupta 2000), turns out to be a distillation of survey studies. In a recent British critical evaluation of the nursing contribution to qualitative research in palliative care from 1990-1999 (Bailey, Frogatt, Field \& Kirshnasamy 2002), the authors found that little attention has been paid to qualitative research in palliative care. They cite only one previous review, Wilkes' 1998 review of nursing research papers-and these were not selected based on methodology, but rather only base on the research focus area (Froggatt, Field, Bailey \& Krishnasamy 2002). Wilkes earlier review takes a quantitative approach to reviewing the evidence. Being the seminal work, perhaps the use of numbers of publications, categorisation of subjects of research, and a breakdown of the quantitative/qualitative divide are helpful in constructing an overview of the field. The author's claim that nurses "are conducting more quantitative research on patient outcomes" is, however, disappointing from a qualitative standpoint.

Froggatt et al (2002), on the other hand, focus their review on "trends across the decade, trends across journal types and the characteristics of (the) sample of papers with respect to the researchers writing (the) papers, the research methodology, research methods adopted (and) the settings and research participants used" (2002: 8). The authors conclude that there is an apparent academic-practice divide and that genuinely collaborative qualitative research would strengthen the applicability of findings for practice (2002: 13). Groundbreaking as these studies are, none mention ethnicity, ethnic minority or diversity in the approaches to end-of-life issues reviewed. Ironically, of the three studies cited above, two include authors with non-European sounding names.

Another review appearing in 2002 (Mitchell 2002) systematically evaluated the literature to answer the question, 'How well do general practitioners deliver palliative care?' Again, ethnicity/race/diversity is overlooked in this examination. The author searched Medline and PubMed databases from 1966 to 2000, identifying 135 references. The conclusions reached included that patients appreciate the contribution the GP makes to palliative care, but reports from relatives suggest that palliative care is performed less well in community settings. GPs expressed discomfort with their competence in this area and felt that appropriate specialist support and facilities were necessary. The study concluded that more is effort is needed by specialist services to develop formal involvement of GPs in the care of individual patients.

A US qualitative review (Teno, Casey, Welch, Edgman-Levitan 2001) of existing professional guidelines for end-of-life medical care was innovatively combined with data from the authors' six focus groups with bereaved family members. By means of this synthesis, the investigators were able to develop a foundation for patientfocused, family-centred end-of-life medical care and to develop a new tool for surveying bereaved family members (2001: 738). The use of family perspectives is important to work on end-of-life care, particularly in studies that include ethnic minorities and diverse populations. Teno et al comment on the difficulties and burdens families face in advocating for the best care for loved ones and the inequalities faced in interactions with the "medical world" (2001: 745). This issue 
becomes particularly important for ethnic minorities in similar contacts and exchanges. The survey tool developed by the authors includes several areas of particular importance for minority respondents: Emotional support; Promoting shared decision-making; Communication, Focus on the individual, Closure; Attending to the needs of family; Family emotional support; Family spiritual support; and Information and education (2001: 746-748).

A whole host of interfaces between the state, the society, the socio-medical culture, the family and the individual come into focus in a particularly poignant way around issues of dying and death. Issues of the right to die, patient self-determination and advance directives and living wills have been on the public agenda for well over a decade in the US. Years of bitter controversy, ethical debates and legal battles preceded the 1991 passage of the Patient Self-Determination Act in the US (Baker 2002: 29). These issues are beginning to come to the forefront of legal, health and ethical debates in the UK as well.

An example of shifting tides in end-of-life care in Britain, the Hammersmith Hospitals NHS Trust in West London recently announced that it would be giving elderly patients a form called an Expression of Wishes in Healthcare (BBC News 2003: 1). This is being done in order to allow patients to tell doctors when to stop treatment if they become so ill that they could not express their wishes. Movements such as these, however, re-enforce the widespread Western concept of the inalienable rights of the individual, but do not take into account the often quite different constructs of the individual in a multiplicity of cultures as well as the sometimes-contrary wishes of family members. As the UK becomes more culturally diverse, the risk to minorities of poor end-of-life care due to cultural misunderstandings is likely to grow just as it has in the US (Krakauer et al 2002: 184). The rights of families to medical knowledge and their roles in decisionmaking are just as valid, inalienable and crucial to the cultural belief systems of many ethic minority communities as are Western patient autonomy models. (See below).

In view of these recent developments in the UK, Baker's (2002) review on ethnic influences on end-of-life decision-making in the US becomes valuable. Although the literature is sparse, Baker's review finds that minority populations complete advance directives less frequently than white populations and minority subjects tend to indicate that they do indeed want aggressive, life sustaining medical interventions administered (2002: 33). For example, a study that compared Asian Gujarati and white elderly persons found that white elderly persons were far more likely to have advance directives and that ethnicity remained significant. Collective research findings consistently suggest that choices involving end-of-life medical treatment decisions may be more related to ethnicity and culture than to age, education, socio-economic status, or other variables (2002: 34). Other important findings in the research on advance directives reviewed include pointing out the fact that an assumption is made that all people are literate, have no untreated vision problems and that patients respond in an individualistic and autonomous manner of thinking as opposed to a collective manner. In actuality, many minority group members may prefer to include and trust their family to make decisions for them (2002: 35). Other factors not often studied also include religiosity and lack of trust in the medical community that could contribute to ethnic differences as well. 
Cultural values and beliefs that are just beginning to be uncovered may well contribute to the reluctance of many people of colour to execute advance directives (2002: 37).

In their study on barriers to optimum end-of-life care for minority patients, Krakauer et al (2002) point to the fact that African Americans prefer aggressive life-sustaining treatment more often than European Americans. The authors discuss issues of mistrust and medical racism, elaborated in their review, as contributing factors. They warn, however:

The literature often construes mistrust as a characteristic of minority patients and populations. This implies that mistrust is a problem that minorities must overcome. It blames the victims of discrimination and racism for a normal response to being victimized. . . The onus should be on physicians and on the healthcare system to consistently demonstrate trustworthiness, not simply on mistrustful minority patients to try to overcome their misgivings.... Physicians first must recognise that the basic values, principles and assumptions of western medicine and bioethics are themselves historically situated and culturally determined" (2002: 186).

In reviewing the reviews of the qualitative literature on end-of-life in regard to ethnicity, race and diversity, two conclusions are drawn. First, the approach to reviews-'systematic' or not-are as diverse as the subject. Passion for the subject under discussion appears to make more convincing arguments than adherence to strict systematic review protocols. Secondly, the subject of race/ethnicity/diversity is just beginning to appear on the agenda in end-of-life studies, most of which are from the US.

The reviews covered here, from several English-speaking countries, range as follows: 1. A synthesis with a view to providing evidence and practical guidelines while defining terms and limitations (Werth et al 2002); 2. A review of the literature of existing professional guidelines combined with data from focus groups of service users to develop a survey tool (Teno et al 2001); 3. A purported qualitative systematic literature review of patient preferences (Higginson \& Sen-Gupa 2000) that resorts to the use of matrixes of mainly survey studies; 4. A systematic review of how well general practitioners deliver palliative care (Mitchell 2002) that concludes that GPs feel inadequately prepared for their interactions in this area; 5. A review of the nursing literature from 1987 to 1996 that describes trends and reflects on a range of both quantitative and qualitative studies (Wilkes 1998); 6 . Two papers on qualitative research in palliative care-one self-titled a descriptive review (Bailey et al 2002) and the other, a critical evaluation (Froggatt et al 2002) that concludes that although well written, nursing papers need improvement in methods-related issues; 7. Finally, one paper (Baker 2002) that reviews the literature during the decade following the Patient Self Determination Act (passed by the US Congress in 1991), concentrating on economic, political and ethnic influences on end-of-life decision-making. 
The paper that, in the estimation of this reviewer, holds the most promise for interpolation into policy and practice in the UK, is one written by three US MDs, Krakauer, Crenner and Fox (Krakauer et al 2002). Reviewing over 150 papers, the authors avoid the pitfalls of becoming bogged down in the review process itself and provide thoughtful discussion on the barriers to optimum end-of-life care for minority patients. Although it is biased in terms of an American point-of-view, the authors present passionate arguments and strong evidence, which are translatable to a British multicultural population. Their discussions of cultural difference, mistrust and medical racism clear the way for meaningful discussions on culturally sensitive end-of-life care for both researchers and policymakers in the UK.

\section{The 'cookbook' approach to diversity}

The cookbook approach to ethnicity/race/diversity and end-of-life issues is often the first response to meeting the educational needs of health and social service workers when interfacing with 'new' cultures. "The majority of published literature contained in medical or nursing libraries or located by searching in health-related bibliographic databases under headings such as ...the names of major religions ... frequently are purely descriptive, or assertive, stating the major principles of the religions concerned and seeking to spell out some the implications of these for practice .... . In the process, they may also create new myths or stereotypes, and compound this with inaccuracies or misunderstandings (Johnson \& Jones 2002: 1). Nonetheless, the 'How To' literature abounds: a set of examples can be found in Green's Nursing Times 1989 to 1992 series on 'Death with Dignity' that describes 13 religious belief systems and their approaches to dying and death as well as one paper on how to transport a body abroad for a funeral. The series is recapitulated in Green's (1993) compendium, "Death with dignity - Meeting the needs of patients in a multi-cultural society."

In Brownlee's 1978 paper on 'The family and health care: explorations in crosscultural settings', the author "discusses what a social worker or health practitioner should know about the family and its influence on health care among various ethnic groups, focusing first on basic information on family organization and interrelationships and then on the family's role during health care. The material is presented in a format designed to be useful to the practitioner in the field, discussing what important aspects of various topics the worker might investigate, why the information is important, and how to gather it" (Brownlee 1978: 179).

In 1987, the British Medical Journal literally produced a "How to Do It" series, described as "A collection of useful advice on topics that doctors need to know about but won't find in the medical textbooks" (Black 1987: 538). Black produced an article for the series of advice on bereavement in certain ethnic groups in Britain that covers Hinduism, Sikhism and Islam and includes religious organisation, imminent death, after death, funeral arrangements, mourning, necropsies, organ donation and transplants, termination of pregnancy, prenatal testing, still births, and deaths of young children in each faith group". Black reached the odd conclusion that in Hindu, Sikh and Islamic communities, "I have not received the impression that there is a need for bereavement counsellors" (1987: 539). Primers are written for paediatricians as well, as represented by Hedayat and Pirzadeh's (2001) overview of the Islamic faith and jurisprudence. The paper 
makes the distinction between Western secular ethics and Islamic emphasis on beneficence over autonomy, particularly at times of death (Hedayat \& Pirzadeh 2001: 965).

One of the problems with the cookbook approach to the study of ethnic minorities is that aspects other than formal religious beliefs are overlooked in much of the "how to' or cookbook literature and not all members of an ethnic group will routinely follow the beliefs of a specific faith (see Mitty 2001). There is a wide variation of beliefs and behaviours within any ethnic population (Kagawa-Singer 2001: 2994). For example, other factors such as folk beliefs and folk medicine are important to many ethnic minority patients and their families. (See Pachter 1994, 'Culture and clinical care. Folk illness beliefs and behaviors and their implications for health care delivery'.)

The cookbook approach also fails when consideration of variations within a specific ethnic group come into focus or generational or cohort differences are considered. Ethnic differences, in many ways, reveal only the outer layer, one skin of identity, transfixing the person underneath. Ethnic differences are structural variables that often obscure equally important building blocks to understanding which include time effects such as age and the life course, cohort effects and generational differences, and historical trends and period effects (Miller 2000: 24). It is crucial throughout any considerations to keep in mind that "individuals and groups can and do change their ethnic or cultural identities and interests through such processes as migration, conversion, and assimilation or through exposure to modifying influences" (Smedley 1993, cited in NCDDR 1999: 1). In the end, cookbook approaches to issues of ethnicity and race do a disservice to both the diverse groups studied as well as the research community as a whole and should be used with caution. "In the light of changing cultural fashions, and variations between individuals in the observance of their faith" (Johnson \& Jones 2002: 2), reliance on such guides is risky.

\section{Issues of faith -other ways forward in the literature}

A better approach to end-of-life issues and faith or spirituality is found, for one example, in the hospice movement and its goal of facilitating mental and spiritual preparation for death. Thibault (2003) makes a case for the "meaning-making" aspects of death and dying in the hospice approach through the development of competency by health care professionals to attend to the age and ethnic diversity, psycho/social/spiritual needs, end-of-life preferences, and ethical and legal concerns of their patients (Thibault 2003: 2). "Meaning-making" helps patients with the goals of remembering, reassessing, reconciling and reuniting (2003: 3) —all goals which are translatable cross-culturally.

Through an interpretive research synthesis of two decades of qualitative studies defining spirituality, Raholm, Lindholm and Eriksson (2002), writing in the Australian Journal of Holistic Nursing, developed a holistic interpretation of spirituality through an inductive process based on comparison. Four themes emerged: undemanding communion; confirmation of dignity; the dialectic of suffering; and reconciliation (2002: 11). The consistencies in these two more thoughtful studies coalesce around Gergen's (2002) constructionist views of the realm of the sacred as a social construction and social constructionism's meaning-making purpose. The 
generative processes that contribute to relationship come into focus in maters of spirituality and death and could be enhanced by dialogue amongst traditions. "The primary question is how do scientific and spiritual discourses (and practices) function within our relationships; what are the reverberations for our lives together here and now and beyond? ... These dialogues should be open to a multiplicity of evaluative criteria. In this way we remain responsible to the very process of meaning-making itself" (2002: 8). It is in this space of wonderment, the unfathomable, the impenetrable source, the sublime-all resistant to logical thinking and commanding our awe-that matters of faith and social construction begin to merge. Through the hermeneutic philosophical position of a commitment to understanding and meaning-making, the relational in dialogues between cultures, faiths, ethnicities and races can take place, enriching societal practice.

\section{End-of-Life decision-making in the literature}

An American qualitative study (Moore \& Sherman, 1999) on factors that influence elders' decisions to formulate advance directives interviewed nine minority and eleven white low-income older people in the community. In reviewing the literature, the authors found that although a large majority indicated that completion of advanced directives is desirable, actually completing medical directives remains low (1999: 23). Those who choose to execute advance directives tend to be white and well educated, largely a middle-class phenomenon; the authors note that religious affiliation/religiosity is a factor (1999: 24). The research examines personal and family systems that influence these decisions. One issue uncovered is that race appears to be related to the degree of communication between parents and adult children regarding end-of-life treatment preferences (1999: 33). Moore and Sherman find that, particularly for minority participants, end-of-life decision-making is couched in religious context, with faith and trust in God and mistrust of human medical technology coupled with a strong belief in an afterlife, making end-of-life planning more comfortable and desirable (1999: 36). In another US qualitative study of mostly white women, however, Carrese, Mullaney, Faden and Finucane ((2002) found that elderly people are resistant to planning in advance for a hypothetical future, particularly for serious illness when death is possible but not certain (2002: 1). The authors found that elderly housebound patients held a worldview that included living life a day at a time (2002: 4).

An end-of-life decision-making study using in-depth narrative interviews and phenomenologic analysis to identify medical decision-making from the perspective of elderly individuals was carried out by Rosenfeld, Wenger and Kagawa-Singer (2000) in the US. Although the ethnic and racial homogeneity of the study sample present limitations, the conclusions add to the debate on a model of dual authority identified in the study. The model contrasts with historical ones for end-of-life decision-making which embrace paternalistic perspectives and contemporary legal standards that do not grant any formal authority to physicians. The authors' data suggest that patients' goals may be achieved best by a model of collaborative surrogate decision-making by families and physicians (Rosenfeld et al 2001: 624). This is particularly interesting in view of other studies that suggest white patients want autonomy, while ethic minority patients do not. This model seems to be in agreement with ethnic studies on patient preferences at end-of-life that indicate more involvement in decision-making by families and that medical staff share 
information with families to inform that decision-making process, rather than only with the patient her/himself (See below).

Narrative method was used in another study (Frank, Blackhall, Michel, Murphy, Azen \& Park 1998) as a follow up to a large survey to explain contradictions in the survey data on end-of-life decision-making among Korean-Americans. The researchers posit that "the reigning conceptualization of autonomy is limited and illusory, and that social relationships may present an equally and sometimes more compelling framework for bioethics than individual rights" (Frank et al 1998: 404). Their use of an individual case study as a follow up to a survey made it possible for the researchers to use narrative to explain an otherwise incomprehensible contradiction in the survey data: Korean Americans report extremely negative attitudes about the use of life-sustaining technology for themselves, but remain positive about its use generally (1998: 406). The subject of the case study interview, a 79 year-old Korean American woman, was consistent in her belief that it is necessary to avoid futile medical treatment while giving control to her family members, preferring that they make any decisions when the time comes (1998: 411). Her story supports the case that a family-centred approach to medical decision-making is part of a broader Korean cultural pattern.

Concepts of "cultural pluralism" and bioethics are raised in a study (Davis 1996) examining the end-of-life decisions made by or for patients who are ChineseAmericans, Black-Americans, Hispanic-Americans and Anglo-Americans. Interviews and observation in a cancer clinic are used to ask whether, in an ethnically diverse culture, we need to reflect on ethical absolutes and ethical relativism (1996: 429). At the core of this study are fundamental differences as to what should be done as defined by the patients/family on the one hand and the healthcare providers on the other. Questions of disclosure or non-disclosure of diagnosis frame the discussion around what is the "ethnically and culturally right thing to do" (1996: 430). "Definitions of both quality of life and good clinical practices about truth-telling were at odds with the conventional western ideas of self-reliance, self-expression and control. Another philosophy in direct opposition to western principles, such as individualism, was adhered to by patients, especially those who are Asians, who maintain that 'self' is embedded with a network of social relations" (1996: 431). The authors conclude that it is vital to raise questions about bioethics that fail to address the reality of cultural pluralism: "Are socially and politically significant factors - such as gender, race, and ethnicity - morally relevant in setting public policy? How can the goal of equality be reconciled with the reality of difference?" (1996: 431).

These questions are further developed by this reviews emerging question, 'What is the family's place in ethical discussions of end-of-life decision-making?' Clearly, Kagawa-Singer is right when she states: "Death creates chaos. It creates a total upheaval in the entire family structure" (1998: 1752).

Bowman, Martin and Singer (2000) developed a taxonomy for quality of end-of-life care viewed from the perspective of patients and their families in communication with their physicians (2000: 54). The authors believe that what is missing from endof-life care is the more in-depth perspective of patients and families: "In recent years, there has been a profusion of measurements of quality health care and of 
end-of-life care specifically. However, selection and application of these measures becomes difficult when values, experiences and preferences of patients and families are largely unknown" (2000: 55). Included in the authors' recommendations for improvements are greater screening and improved response time for spiritual and psychosocial support. They develop an alternative: a semistructured interview guide with open-ended questions in the areas of advance care planning, spiritual/psychosocial support and pain and symptom control. Qualitative analysis is applied, highlighting families' attitudes; the authors point out that persons from different ethnic backgrounds may differ substantially in the value they attach to quality indicators. Cultural differences remain central to these measures and interviewers proficient in the appropriate language are used (2000: 59).

\section{Lessons from some other cultures: Israel, Spain \& Sweden}

A qualitative study using a hermeneutic phenomenological method of analysis explores the values underlying end-of-life decisions in Israel (Lechtentritt \& Rettig 2001). This is an interesting group to study because of the communitarian framework of Israeli society with its high degree of collective consciousness, solidarity, belonging, mutual concern, and interdependence. This collective worldview is often the overriding norm in Israel when addressing end-of-life decisions (Lechtentritt \& Rettig 2001: 151). Through a case study approach used to discuss moral issues, the Israeli social context is identified as an important contributor in establishing individuals' values toward end-of-life decisions. Quality of life and quality of death are intertwined, an understanding that is often missing in the quality-of-life literature (2001: 158). This study is among the first to examine explicitly the values underlying end-of-life decisions.

A Spanish study (Olarte \& Guillén 2001) presents key ethical issues in palliative and end-of-life care in Spain and how these issues are influenced by Spanish culture. The authors compare the distinct nature of the bioethics school of thought in Spain with that in the US. They make note of the cultural remnants of previous religious attitudes influencing health care and palliative care and how these differ from Protestant ways predominant in North America or Northern Europe where internal locus of control (or patient autonomy) is the norm (2001: 48). The authors point out that, in Spain, as in all other predominantly Catholic countries, the term 'hospice' has negative connotations, denoting extreme poverty or orphanage.

The present General Health Law in Spain allows for communication of prognosis to family members and withholding of information to patients, "for the benefit of the patient, although this approach should be considered exceptional..." (2001: 52). For instance, the term 'cancer' is frequently omitted when the patient is present at Spanish consultations and a cancer diagnosis is not always transmitted to patients. A majority of Spanish families are opposed to informing the patient, which largely determines the information given to the patient (2002: 51). The authors conclude:

Spain is not alone in the "cultural continent" gradually emerging in the English literature confronting the Anglo-Saxon "cultural continent" (United Kingdom, United States, Northern Europe, Canada, Australia, and New Zealand) that up to now has provided the majority of patients mentioned in the literature in English about truth-telling. Studies in Italy, Greece, the 
former Soviet Union, Latin America, Japan, and the Philippines report results similar to Spanish results. (2002: 51)

Sweden has changed in the past few decades from a society with a few ethnic groups to one with over a hundred (Ekblad, Marttila \& Emilsson 2000: 623). Ekblad et al's qualitative focus group study on cultural challenges in end-of-life care concludes that to better understand other cultures it is important to raise awareness about staff's own culture and to pay attention to culture, especially in the context of the individual. This context includes attention to issues of health care systems as cultural constructs, that beliefs about health and disease are culturally determined, that even within specific cultural groups variances exists, and that death and disease are inevitably understood and experienced within a complex web of cultural meanings (2000: 624). Interestingly, in this study "the staff found that using a relative as interpreter can lead to complications; sometimes relatives function well as interpreters, but they may interpret selectively leaving staff unaware of whether all the patient has said has been translated. The relative may have omitted things he/she did not want the patient to bring up" (2000: 627). It could also be a case in reverse that the relative's belief system includes keeping certain information from the patient, as is evident in many of the studies under review. The authors in this study recommend an approach with patients nearing the end of life and their families that includes evaluating each patient and family, rather than memorising traits associated with different ethnic groups (2000: 629).

\section{Tools}

The Toolkit of Instruments to Measure End-of-Life Care, dubbed the toolkit project, was started in the US in 1996 to outline and assess new measurement tools that can be utilized as part of audits to describe and asses the dying experience (Teno, Field \& Byock 2001: 714). Although beyond the qualitative brief of this paper, the development of measurement tools through rigorous use of qualitative methods is noteworthy. The various tools are available online at: http://www.chcr.brown.edu/pcoc/toolkit.htm and are linked at the CEEHD website (http://users.wbs.warwick.ac.uk/chess/ceehd/ceehd.htm). Some of the other qualitatively designed approaches to understanding end-of-life issues are discussed below.

Kagawa-Singer and Blackhall (2001: 2993) find that culture fundamentally shapes how individuals make meaning out of illness, suffering and dying. Diversity of both patients and physicians includes the risk for cross-cultural misunderstandings. Kagawa-Singer uses two case studies-an African-American couple and a Chinese-American family to outline some of the major issues involved in crosscultural care to develop techniques for negotiating issues influenced by culture for end-of-life care. Raising issues such as responses to inequities in care, communication and language barriers, religion and spirituality, truth telling, family involvement, and hospice care, the authors raise both the consequences of ignoring these issues as well as techniques and strategies to address them. The techniques are generic in approach and, therefore, valuable to translation into a wide variety of cross-cultural settings. A second tool is developed to ascertain the level of cultural influence in end-of-life decision-making that includes attitudes of patients and 
families, beliefs, context, decision-making style and environment. The tool first seeks out relevant information in each category (e.g., 'What attitudes do this ethnic group in general, and the patient family in particular, have toward truth telling about the diagnosis in progress?') followed up with questions and strategies (e.g., 'What is the symbolic meaning of the particular disease?') The authors point out that, since December 2000 in the US, cultural competency is not simply a moral or ethical obligation, it is the law and that there are now national standards for culturally and linguistically appropriate health services (2001: 3000).

In a proposal of a model for evaluating the quality of dying and death, Patrick, Engelberg and Curtis (2001) cite six conceptual domains derived from qualitative data analysis: symptoms and personal care, preparations for death, moment of death, family, treatment preferences, and whole person concerns. These domains encompass 31 aspects that can rated by patients and others as to their degree of importance prior to death and assessed by significant others or clinicians after death to assess the quality of the dying experience (2001: 717). The study stands out in that it is one of a few to directly compare the account given by dying persons with their loved ones or caregivers (2001: 719).

The ETHNIC(S) Mnemonic is a clinical tool and framework for practioners to use in providing culturally appropriate geriatric care (Kobylarz, Heath \& Like 2002) in response to US legislation to deliver culturally responsive services to people of diverse racial, ethnic and cultural backgrounds. The authors caution that diversity is often greater within than between groups in terms of health beliefs, attitudes and perspectives on the delivery of health care (2002: 1582). The tool is pragmatic and integrated into the routine 15-minute visit between physicians and their patients in the ambulatory office setting, the hospital and other ambulatory settings (2002: 1583). The domains of ETHNIC(S) are: explanation, treatment, healers, negotiate, intervention, collaborate and spirituality (seniors). Some of the issues raised under various domains include, amongst others, the passive roll that some elders may automatically assume in decision-making; that healthcare providers from one cultural background may have limited awareness of the media sources used by patients from different cultural backgrounds; and that older patients from varying cultures traditionally use complementary and alternative medicine treatments that need to be explored.

Dowd, Poole, Davidhizar and Giger (1998) further develop Giger and Davidhizar's nursing assessment model to provide culturally relevant death education and grief counselling. The authors champion pragmatic and workable models, instead of 'facts' applied in isolation (1998: 35). Using examples from a wide variety of global cultures, Dowd et al include an Appendix of cultural implications of various cultural and religious groups in the death and dying process and a pre-test/post-test for the use of educators.

Murray, Boyd, Kendall, Worth, Benton and Clausen (2002), in a UK study develop guidelines for end-of-life care through qualitative interviews with patients with either lung cancer or cardiac failure, their carers and professional carers. They conclude that care for people with advanced progressive illness is currently prioritised by diagnosis rather than need and that care should be proactive and designed to meet patients' specific needs. Supporting the WHO (World Health Organisation) 
palliative care approach, the authors proposed solutions to information needs of patients, strategic planning across disciplines, management programmes that identify people who 'reasonably might die', training for professionals in communication skills, specialist palliative care advice available for patients with advanced non-malignant diseases and training for such specialists (2002: 4).

\section{Grey Literature and Web-based Knowledge}

Grey literature and web-based knowledge and their inclusion in systematic reviews are swiftly becoming the singularly most important methodological contribution to the democratisation being made of the evidence-based movement. Grey literature is comprised of the literature that is not found in peer reviewed journals and is made up of practitioner journal literature, conference papers, books, literature from a range of public, private and voluntary sector bodies, and government publications. Increasingly, much of this information is becoming available through the worldwide web, blurring the boundaries between physical literature and electronic manifestations of information. The web-based resources and grey literature uncovered in this review's search are listed in 'Websites of interest to end-of-life researchers and policy-makers' and the 'Annotated Bibliography: Qualitative review of end-of-life and ethnicity/race/diversity', both available at the CEEHD website (http://users.wbs.warwick.ac.uk/chess/ceehd/ceehd.htm).

For example, a study of diversity and end-of-life care from the American Health Decisions position papers (http://www.ahd.org/ahd/library/position/ValuesEnd.html) (Jennings 1999), reviews surveys, community forums and focus group studies on end-of-life decision-making to reach its recommendations. One of the focus group studies included 36 gatherings in 32 cities across the US. The results describe much overlapping consensus across ethnic and religious diversity with a core set of attitudes emerging from the study (Jennings 1999: 6). "By and large, demographic factors such as age, religion, region, or ethnicity made little difference to the content of the focus group discussions..." (1999: 7). Recommendations included the fact that people trust their families and that they should be more empowered to make decisions and better supported in providing care to a dying family member (1999: 7).

Jennings cautions, however, that minority cultural perspectives are generally underrepresented and under-articulated in studies and projects of the type under consideration in his paper (1999: 11). He makes the admonitory point that: "Cultural difference may ... be difficult to express in what feels like a highly secular and western setting. More homogeneous groupings, led by community leaders who are more well known and better trusted by the participants may be required to open up different points of view effectively" (1999: 12). Jennings concludes: "The subjects of death, dying, pain, suffering, care, dignity, and peace at the end of life may in fact lead one to that terrain where our diverse humanness recedes and our common humanity comes to the fore" (1999: 12).

In another web-based paper, Ronald K. Barrett reviews two books, Counseling the Culturally Different: Theory and Practice (D. Sue, 1990) and Counseling American Minorities: A Cross-Cultural Perspective (D Atkinson, G. Morten \& D.Sue, 1989) on the American Medical Association website (http://www.ama- 
assn.org.ama/pub/category/6824.html). In his review entitled, 'Recommendations for Culturally Competent End-of-Life Care Giving', Barrett synthesises recommendations from these two books. Issues discussed include: stereotyping, cultural self-awareness including biases and value systems regarding care, comfort with multicultural differences in approaches to care, willingness to refer to caregivers who share the patients background, institutional barriers that prevent minorities from using care, and understanding and sensitivity to individual and group differences.

The 'Caring for the Dying Poor Project', based at Indiana University School of Medicine (http://shaw.medlib.iupui.edu/ethics/dyingpoor.html) and funded by the Open Society Institute, gathered descriptive, qualitative data on patients, families and care providers. It finds that the conditions of poverty and race significantly shape the end of life experience. The Project also finds great divergence between the point of view of providers and the community they serve. The Project developed, 'Uncovering the Hidden Faces of the Dying Poor', a home-based education programme for first and third year medical students and residents with a palliative and psychosocial focus.

In a short report to help medical professionals see how race colours end-of-life issues, Goodkind describes African-American La Vera Crawley's experience with her mother's entry into hospice weeks before her death. Crawley explains: "Caregivers should understand that African-Americans often view palliative care from a tradition of struggling against death that dates back to the times of slavery and segregation, as well as from a predominantly Christian background that has roots in African religions" (Goodkind 2001: 1). For instance, Crawley states that she was taken aback when a white nurse suggested that her mother's quality of life had deteriorated to an extent that death might be preferable. Crawley explains, in the African-American community, the need to struggle can overshadow concerns about quality of life: "African-Americans feel they must cling to life whenever it is present" (Goodkind 2001: 2).

The Ian Anderson Continuing Education Program in End of Life care (http://www.cme.utoronto.ca/endoflife/) is a joint project of Continuing Education and The Joint Centre for Bioethics, University of Toronto and The Temmy Latner Centre for Palliative Care, Mount Sinai Hospital, Toronto. Its goal is to educate 10,000 primary care physicians and specialists across Canada over a five-year period to deal with issues surrounding death and dying. It also holds conferences relevant to dying in critical sectors including healthcare institutions and educators, media and government.

The lan Anderson Program consists of nine teaching modules: Palliative Care Standards and Models, Pain Management, Symptom Management, End-of-Life Decision-Making, Communications with Patients and Families, Psychological Symptoms, The Last Hours, Culture and Conflict Resolution. The website includes resources and the educational modules in PowerPoint-type layout available in pdf format. Of particular interest to this study is the module, 'Culture in End-of-Life Care' (http://www.cme.utoronto.ca/endoflife/PPT\%20Culture.pdf). 
The UK Department of Health's 'No Exclusion Clause Project: Opening doors to better palliative care services for people from culturally diverse communities' (http://www.doh.gov.uk/race equality/noexclusion.htm) is introduced on the Department's website. The project was taken forward by a series of focus groups to obtain information on the current quality and accessibility of palliative care services. A community facilitator spoke directly to minority ethnic groups in their own cultural environments. Six major failings in palliative care were uncovered: lack of appropriate information, communication barriers, difficulties accessing professional interpreters, cultural and religious influences that can hinder access, stereotyping, and poorly organised and rarely available training to help professionals address diversity, cultural values and health beliefs. Development of training for professionals and information for cultural communities in video format were key suggestions from the study.

The website, Innovations in End-of-Life Care (http://www2.edc.org/lastacts/), is an international journal and online forum of leaders in end-of-life care with a searchable database. Of particular interest is the paper, 'International Perspective' -an interview with Henry T. Dom on Vaisnava Hindu and Ayurvedic approaches to caring for the dying (http://www2.edc.org/lastacts/archives/archives Nov99/intlpersp.asp). Dom is a practioner of the ancient Vaisnava tradition of Hinduism. His perspective on the place of spirituality in palliative care is rooted in his own multi-cultural, multi-lingual background in India, South Africa and the UK where he worked with the elderly and those dying from Aids, cancer and other diseases in nursing homes, hospitals and hospices. Dom particularly emphasizes the need for Western hospitals to be sensitive to the needs of non-Christian patients by involving the family in the physical care of the patient, making the care environment homelike, accommodating dietary needs, allowing or encouraging patients and families to engage their own spiritual leaders in care, and making the care environment less institutionalised overall.

The National Council for Hospice and Specialist Palliative Care Services (http://www.hospice-spc-council.org.uk/) is the umbrella and representative body for hospice and palliative care in England, Wales and Northern Ireland. Its brief includes: representation of the collective views and interests of hospice organisations and palliative care services to ministers, civil servants, members of parliament, the media and other key statutory agencies in England, Wales and Northern Ireland and collaboration with the Scottish Partnership Agency for Palliative and Cancer Care; developing and maintaining a UK perspective across palliative care develop policies to promote better collaboration and co-ordination between the voluntary, health and local authority sectors and to identify and promote additional funding requirements at all levels; developing professional standards and clinical guidelines in association with others with an interest in this work, taking on board findings on patient perspectives; encouraging improved professional education and research in all aspects of palliative care, working closely with universities and colleges, the medical and nursing Royal Colleges and international agencies; publishing regular information about the work of Council, those it represents and the individuals and agencies with which it liases; and publishing clinical guidelines and papers on palliative care issues such as quality, clinical governance, commissioning, partnership working, ethics and research. 
Of particular interest to this study are several Council publications (http://www.hospice-spc-council.org.uk/publicat.ons/publicationslist.htm) that deal specifically with palliative care and ethnic minority communities-particularly those papers that have developed from the Council's own seminars. The 1995 occasional paper, 'Opening Doors: Improving Access to Hospice and Specialist Palliative Care Services by Members of the Black and Ethnic Minority Communities' (Hill and Penso, 1995) laid the groundwork for study of palliative care in this area in Britain. The authors identified several factors that contribute to the perceived low uptake of specialist palliative care services by members of minority communities. In reviewing that study in 'Palliative Care Services for Different Ethnic Groups' (Mount, Ed, 2001), Firth comments that "Service users were not interviewed for the 1995 project, and it did not address the different needs of different black and ethnic minority groups, or recognise the differences existing between groups" (2001: 4). Firth also points out that the bulk of the studies refer to people from the Indian subcontinent and the Caribbean, but there are little or no data on Chinese, Arabs, Turks, Greeks and new immigrants. She concludes that palliative care service provision is based on existing Western models of care (2001: 4).

Firth continues: "The use of terms like 'black and ethnic minority communities' highlights a real dilemma which is reflected in the language and terminology used in much of the medical and nursing literature in the UK. In the US, Canada, Australia and New Zealand the emphasis is on multiculturalism, pluralism and diversity" (2001: 4).

'Ethnic Minorities' may be of value when high-lighting discrimination, inequity and racism, and in this sense it is a political term. Certainly, at the level of provision and policy these issues must be emphasised. But there is also a danger that as long as we refer to 'minorities' we tend to distance ourselves, and assume implicitly that both we 'British', and 'they', the 'Black and Ethnic Minorities', are monocultural or homogeneous. This does not acknowledge the very great diversity among different ethnic minority groups, including 'white' Britons... The use of the expression 'black and ethnic minority may be useful in a political context but it is also in danger of creating a sense of otherness. (2001: 5).

In another paper from the seminar, Simmonds, speculating from census data, proposes that as younger cohorts of Black and Asian British age and second and third generation British Asians follow a more Western way of life, the need for specialist palliative and hospice care services will actually increase for these ethnic groups (2000: 7-8). The author concludes that face-to-face contact between the hospice and first generation Asians might prove more effective than written information, even when it is offered in a variety of languages (2000: 9). 


\section{Development of models through synthesis and integration: \\ End-of-life decisions in alternative life trajectories-the case of Aids}

When writing/reading of end-of-life or dying and death we all engage at some point in the personification of the subject(s) with whom we are engaging second-hand. Typically, we try to banish this process to the back of our minds, professionally. Still, images and remembrances of our parents, grandparents or others who's dying process we have experienced personally come to the fore in the exercise. It is not uncommon, when reading about end-of-life concerns, to picture someone, typically old and frail, who's death we may have experienced personally. Instead of ignoring this, a post-modern approach would ask us to reflect upon it and find out how this phenomenon might be helpful in our work. "Many modern cultures have a knack of avoiding the emotional reality of death unless they are forced to confront it. ... Emotional involvement by the researcher is only restricted to the extent that it prevents the researcher from having a clear awareness of what is truly going on or clouds the reality" (Mishara 1993: 92-93).

On the other hand, by thinking of death and dying only in terms of people who are coming to the end, with long lives behind them and approaching 'good deaths', we avoid personally reflecting upon cases of 'bad deaths' or unanticipated deaths, including those that may be the result of alternative and/or diverse life trajectories.

Death from Aids, typically a premature death, is one of those cases. The amount of qualitative literature on end-of-life and Aids has grown exponentially since the beginning of the pandemic in the 1980s; the literature on cases from racial and ethnic minority populations has developed as well. This review will not include endof-life studies and Aids, except to make a few points and recount some lessons about integration and synthesis of practices models learned from the literature.

A watershed was reached in the Aids pandemic (in the late 80s) when more people knew of a family member, friend, colleague or acquaintance that was HIV+ or had died from Aids than those who did not. Personal experience of knowing someone with HIV+ or Aids contributed greatly to the reduction of prejudice in society and the ability to move knowledge forward. It was, in part, through this process of 'personification' of the disease that this progress was made. The realization that a segment of the population was being devastated by the disease also contributed to a consciousness raising and a rallying around what became a socio-political event in the context of collective loss within communities.

Research on HIV+ or Aids, particularly in the British literature, came into the mainstream of care literature in the late 1980's and early 1990's. It was care for the elderly, particularly care for the elderly in the community, that first informed Aids service planners and providers of care possibilities, forming the basis for new structures of community care for people with HIV+ or Aids (Jones 1992). The HIV+ and Aids service provision sector took up what was currently best practice in community care and synthesised and integrated it. In the early scramble to provide care systems for those affected by the disease, many innovative and groundbreaking approaches to care were developed by medical, social and voluntary agencies through this process. This period was also the initial period of 
growth of the hospice movement in the UK. Much has been learned from Aids service provision about people's ability to meet the challenges of care, particularly in the face of negativity and prejudice and ways to develop new practice through synthesis and integration of older models.

Community care for people living with HIV+ or Aids is important to the considerations here because it presents a dynamic, amorphous model for the development of end-of-life care. Typically, the continuum of care for persons living with HIV+ or Aids often first includes a reliance on friends and partners in the early stages of the disease for minimal care and support (Folkman, Chesney \& Christopher-Richards 1994: 50). This is often followed by an expansion of the care network to include volunteers and health professionals as complications increase and, finally, concludes with a late and often painful decision to involve and rely upon family members for belated emotional support and terminal care provision (Jones 1992: 15). This point is made in the larger discussion here because the need to return to family, even in situations where reconciliation with the family is a necessary part of the process, is common in end-of-life case histories in Aids studies. As part of this process, it can be substantiated that the need for family to be involved in end-of-life decisions is prevalent in cases of Aids, even when alienation from family members has preceded it. Early Aids cases typically occurred in minority populations in Western countries, (including gay people, intravenous drug users and immigrants from African cultures). The conclusion is drawn, therefore, that family involvement in end-of-life decision-making cannot be ignored and that persons from a wide range of ethnic and/or racial backgrounds (including the white population) prefer family involvement. These parallels in more general end-of-life care, particularly for people from diverse and minority communities, cannot be ignored.

\section{Conclusions and recommendations}

This paper has investigated the qualitative literature on end-of-life and ethnicity/race/diversity, using qualitative methods to underpin the review. It champions the usefulness of evidence for end-of-life provider, policymaker and service-user circles in the UK and elsewhere and contributes to the larger society's dialogue on death and dying more generally.

Key findings include:

- The review of qualitative literature on palliative care is minimal, with little mention of ethnicity/race/diversity; palliative care generally pays little attention to qualitative methods.

- As the UK becomes more culturally diverse, the risk to minorities of poor end-of-life care due to cultural misunderstandings is likely to grow just as it has in the US.

- The rights of families to medical knowledge and their roles in decisionmaking are just as valid, inalienable and crucial to the cultural belief systems of many ethic minority communities as are Western patient autonomy models. 
- Collective research findings consistently suggest that choices involving endof-life medical treatment decisions may be more related to ethnicity and culture than to age, education, socio-economic status, or other variables.

- Healthcare providers must recognise that the basic values, principles and assumptions of western medicine and bioethics are themselves historically situated and culturally determined.

Resorting to 'cookbook' approach to diversity creates new myths or stereotypes and compounds this with inaccuracies and misunderstandings, including that the problem that aspects other than formal religious beliefs are overlooked. Not all members of an ethnic group will routinely follow the beliefs of a specific faith. It is important to recognise that building blocks to understanding include time effects such as age and the life course, cohort effects and generational differences, and historical trends and period effects. The meaning-making role of the hospice movement's practice of facilitating mental and spiritual preparation for death establishes a more holistic model for care. The sacred as a social construction is noted as the foundation for a much-needed dialogue amongst cultural traditions.

End-of-life decision-making is often couched in religious contexts. For many faiths and cultures, the giving up of control to family members is more common practice than in some Western cultures. Questions of disclosure of patient information become embedded within a network of social relations in many non-Western and/or non Northern-European cultures. The question of the family's place in ethical discussions of end-of-life decision-making pervades the reviews' studies and these include studies of a wide variety of cultures and faiths.

This preliminary review of the qualitative literature on diversities in approach to endof-life now makes it possible to move the process to a final analysis and review by the use of reflective teams using a narrative interpretive method (Jones 2003) combined with classic concepts of the utility of case study (Yin 1989). This procedure, envisaged as one that would begin with the watershed studies and cutting-edge research uncovered and synthesized in this preliminary review, would be used to inductively reach polyvocal conclusions that could benefit policy and practice. The time has arrived when parochial views, practices and concepts in end-of-life care will no longer suffice in a multi-cultural/racial/ethic global society. If for no other reason, the swift exchange of information and practice now possible on a global scale makes narrow and regional/local methodologies obsolete. Rather than forcing minority practices and belief systems into constricted 'host' country systems, an enlightened worldview is required that approaches diversities in practice as new information beneficial to the wider society as a whole, expanding policy, practice and the collective conscience. "Unless clinical scientists make specific and explicit efforts to understand and tackle the concerns that lead to exclusion or non-participation in research of certain groups in society, ...the research will be fatally flawed" (Johnson 2003: 10). We might add that if society does not make the same sort of effort, it will be flawed as well. 


\section{References}

Anonymous (1999). Vaisnava Hindu and Ayurvedic Approaches to Caring for the Dying:An Interview with Henry T. Dom, Phd (Hari-Dhama). Innovations in End-of-Life Care. Available from http://www2.edc.org/lastacts/archives/archivesNov99/intlpersp.asp

Bailey, C., Froggatt, K., Field, D., \& Krishnasamy, M. (2002). The Nursing Contribution to Qualitative Research in Palliative Care 1990-1999: A Critical Evaluation. Journal of Advanced Nursing, 40(1), 4860.

Baker, M. E. (2002). Economic, Political and Ethnic Influences on End-of-Life Decision-Making: A Decade in Review. Journal of Health and Social Policy, 14(3), 27-40.

Barrett, R. K. (2001). Recommendations for Culturally Competent End-of-Life Care Giving. Available from http://www.ama-assn.org/ama/pub/category/6824.html.

BBC-News (2003). Patients to Decide Treatment End. Available from http://news.bbc.co.uk/go/pr/fr/12/hi/uk_news/england/2793973.stm.

BBC-TV FOUR (2003). Ebony Towers: The New Black Intelligentsia. M. Poole (Producer).

Black, J. (1987). Broaden Your Mind About Death and Bereavement in Certain Groups in Britain. British Medical Journal, 295(6597), 536-539.

Bowman, K. W., Martin, D. K., \& Singer, P. A. (2000). Quality End-of-Life Care. Journal of Evaluation in Clinical Practice, 6(1), 51-61.

Brownlee, A. (1978). The Family and Health Care: Explorations in Cross-Cultural Settings. Social Work in Health Care, 4(2), 179-178.

Calasanti, T. M. (1996). Incorporating Diversity: Meaning, Levels of Research, and Implications for Theory. Gerontologist, 36(3), 147-156.

Carrese, J. A., Mullaney, J. L., Faden, R. R., \& Finucane, T. E. (2002). Planning for Death but Not Serious Future IIIness: Qualitative Study of Housebound Elderly Patients. British Medical Journal, 325(7356), 125-127.

Davis, A. (1996). Ethics and Ethnicity: End-of-Life Decisions in Four Ethnic Groups of Cancer Patients. Medical Law, 15(3), 429-432.

Department of Health (DoH) (2001). No Exclusion Clause Project. Available from http://www.doh.gov.uk/race_equality/noexclusion.htm.

Dowd, S. B., Poole, V. L., Davidhizar, R., \& Giger, J. N. (1998). Death, Dying and Grief in a Transcultural Context: Application of the Giger and Davidhizar Assessment Model. Hospice Journal, 13(4), 33-56.

Ekblad, S., Marttila, A., \& Emilsson, M. (2000). Cultural Challenges in End-of-Life Care: Reflections from Focus Groups' Interviews with Hospice Staff in Stockholm. Journal of Advanced Nursing, 31(3), 623-630. 
Folkman, S., Chesney, M.A., Christopher-Richards, A. (1994) Stress and coping in caregiving partners of men with aids. Psychiatric Manifestations of HIV Disease Vol. 17:1 March: 35-53.

Frank, G., Blackhall, L. J., Michel, V., Murphy, S. T., Azen, S. P., \& Park, K. (1998). A Discourse of Relationships in Bioethics: Patient Autonomy and End-of-Life Decision Making among Elderly Korean Americans. Medical Anthropology Quarterly, 12(4), 403-423.

Froggatt, K. A., Field, D., Bailey, C., Krishnasamy, M. (2002). Qualitative Research in Palliative Care 1990-1999: A Descriptive Review. International Journal of Palliative Nursing, (under review).

Gergen, K.J. (1997) Social Theory in Context: Relational Humanism. Draft copy for J. Greenwood (ed), The mark of the social. New York: Rowman and Littlefield. Available from http://www.swarthmore.edu/SocSci/kgergen1/web/printer-friendly.phtml?id=manu9.

Gergen, K.J. (2001) Construction in Contention Toward Consequential Resolutions. Theory \& Psychology, 11, 3: 419-432.

Gergen, K. (2002). Reflecting on/with My Companions. In C. Hermans, Immink, G., deJong, A. and van der Lans, J., (Eds), Social Constructionism and Theology. Boston: Brill.

Goodkind, M. (2001). Crawley Helps Medical Professionals See How Race Colors End-of-Life Issues. Available from http://www.stanford.edu/dept/news/report/news/september19/crawley.html.

Gramelspacher, G. P. (1999). Caring for the Dying Poor Project. Available from http://shaw.medlib.iupui.edu/ethics/dyingpoor.html.

Green, J. (1989). Death with Dignity: Islam. Nursing Times, 85(5), 56-57.

Green, J. (1989). Death with Dignity. Hinduism. Nursing Times, 85(6), 50-51.

Green, J. (1989). Death with Dignity: Sikhism. Nursing Times, 85(7), 56-57.

Green, J. (1989). Death with Dignity: Judaism. Nursing Times, 85(8), 64-65.

Green, J. (1989). Death with Dignity: Buddhism. Nursing Times, 85(9), 40-41.

Green, J. (1989). Death with Dignity: Baha'i Faith. Nursing Times, 85(10), 50-51.

Green, J. (1989). Death with Dignity: Funerals Abroad. Nursing Times, 85(11), 63.

Green, J. (1992). Death with Dignity--Jehovah's Witnesses. Nursing Times, 88(5), 36-37.

Green, J. (1992). Death with Dignity. Christianity. Nursing Times, 88(3), 25-29.

Green, J. (1992). Death with Dignity. Christian Science. Nursing Times, 88(4), 32-33.

Green, J. (1992). Death with Dignity. Rastafarianism. Nursing Times, 88(9), 56-57.

Green, J. (1992). Death with Dignity--the Mormon Church. Nursing Times, 88(6), 44-45. 
Green, J. (1992). Death with Dignity. Zoroastrianism. Nursing Times, 88(7), 44-45.

Green, J. (1992). Death with Dignity. The Afro-Caribbean Community. Nursing Times, 88(8), 50-51.

Green, J. (1993). Death with Dignity - Meeting the Needs of Patients in a Multi-Cultural Society. London: Nursing Times.

Harré, R. (1997) He lived to tell the tale. Journal of Narrative and Life History 7, 1-4: 331-334.

Hedayat, K. M., \& Pirzadeh, R. (2001). Issues in Islamic Biomedical Ethics: A Primer for the Pediatrician. Pediatrics, 108(4), 965-971.

Higginson, I. J., Finlay, I., Goodwin, D. M., Cook, A., M., Hood, K., Edwards, A. G. K., Douglas, H. R., Norman, C. E. (2002). Do Hospital-Based Palliative Teams Improve Care for Patients or Families at the End of Life? Journal of Pain and Symptom Management, 23(2), 96-106.

Higginson, I. J., Sen-Gupta, G. J. A. (2000). Place of Care in Advanced Cancer: A Qualitative Systematic Literature Review of Patient Preferences. Journal of Palliative Medicine, 3(3), 287-300.

Hill, D., Penso, D. (1995). Opening Doors: Improving Access to Hospice and Specialist Palliative Care Services by Members of the Black and Ethnic Minority Communities (Occassional Paper 7): National Council for Hospice and Specialist Palliative Care Services.

Jennings, B. (1999). Values near the End of Lives:Grassroots Perspectives and Cultural Diversityon End-of-Life Care. Available from http://www.ahd.org/ahd/library/position/ValuesEnd.html.

Johnson, M. R. D. (2003). Research Governance and Diversity: Quality Standards for a Multiethnic NHS. Nursing Times Research, 8(1), 27-35.

Johnson, M. R. D., Jones, K. (2002). Faith \& Health a Critical Review. Leicester: The Leicester Faith \& Health Forum.

Jones, K. (1992) "Dark Elegies" AIDS \& OLDER PEOPLE: The kinship of caring, sharing and loss. MSc. thesis, Age Concern Institute of Gerontology, King's College - London, University of London.

Jones, K. (2003a). The Turn to a Narrative Knowing of Persons: One Method Explored. Nursing Times Research, 8(1), 60-71.

Jones, K. (2003b). Mission Drift in Qualitative Research, or Moving toward a Systematic Review of Qualitative Studies, Moving Back to a More Systematic Narrative Review. Centre for Evidence in Ethnicity, Health \& Diversity (CEEHD). Available from http://users.wbs.warwick.ac.uk/chess/ceehd/ceehd.htm.

Kagawa-Singer, M., \& Blackhall, L. J. (2001). Negotiating Cross-Cultural Issues at the End of Life: "You Got to Go Where He Lives". Journal of the American Medical Association, 286(23), 2993-3001.

Knebel, A. R. (2002). Research Initiatives to Improve End-of-Life Care. Geriatric Times, III(6).

Kobylarz, F. A., Heath, J. M., \& Like, R. C. (2002). The Ethnic(S) Mnemonic: A Clinical Tool for Ethnogeriatric Education. Journal of the American Geriatrics Society, 50(9), 1582-1589. 
Krakauer, E. L., Crenner, C., \& Fox, K. (2002). Barriers to Optimum End-of-Life Care for Minority Patients. Journal of the American Geriatrics Society, 50(1), 182-190.

Kundnani, A. (2002). The Death of Multiculturalism. Independent race and refugee news network, Institute of Race Relations. Available from http://www.irr.org.uk/2002/april/ak000001.html.

Leichtentritt, R. D., \& Rettig, K. D. (2001). Values Underlying End-of-Life Decisions: A Qualitative Approach. Health and Social Work, 26(3), 150-159.

Meacham, J. (1999) Riegel, dialectics, and multiculturalism. Human Development 42: 134-144.

Miller, R. (2000) Researching Life Stories and Family Histories. London: Sage Publications.

Mishara, B. L. (1993). Thanatology Research from Quebec: A Different Emphasis. Omega, 27(1), 91.

Mitchell, G. K. (2002). How Well Do General Practitioners Deliver Palliative Care? A Systematic Review. Palliative Medicine, 16(6), 457-464.

Mitty, E. L. (2001). Ethnicity and End-of-Life Decision-Making. Reflections on Nursing Leadership, 27(1), 28-31, 46.

Moore, C. D., \& Sherman, S. R. (1999). Factors That Influence Elders' Decisions to Formulate Advance Directives. Journal of Gerontological Social Work, 31(1/2), 21-39.

Mount, J. (2001). Palliative Care Services for Different Ethnic Groups Proceedings of a Seminar Held in December 2000: National Council for Hospice and Specialist Palliative Care Services.

Murray, S. A., Boyd, K., Kendall, M., Worth, A., Benton, T. F., \& Clausen, H. (2002). Dying of Lung Cancer or Cardiac Failure: Prospective Qualitative Interview Study of Patients and Their Carers in the Community. British Medical Journal, 325(7370), 929.

National Center for the Dissemination of Disability Research (NCDDR). (1999). The Socially Constructed Nature of Race, Culture, and Disability. Available from http://www.ncddr.org/du/researchexchange/v04n01/concepts.html.

Nickerson, K. (2001). Valuing Diversity: Key Components to Ethical Health Care Admininstration, Service, and Research. Available from http://www.med.howard.edu/ethics/handouts/Diversity\%20Lecture\%20F'01.pdf.

Noblit, G. W., Hare, R. Dwight. (1988). Meta-Ethnography : Synthesizing Qualitative Studies. Newbury Park, Calif. ; London: Sage.

Olarte, J. M. N., \& Guillén, D. G. (2001). Cultural Issues and Ethical Dilemmas in Palliative and Endof-Life Care in Spain. Cancer Control, 8(1), 46-54.

Pachter, L. M. (1994). Culture and Clinical Care. Folk Illness Beliefs and Behaviors and Their Implications for Health Care Delivery. Jama-Journal of the American Medical Association, 271(9), 690-694. 
Patrick, D. L., Engelberg, R. A., \& Curtis, J. R. (2001). Evaluating the Quality of Dying and Death. Journal of Pain and Symptom Management, 22(3), 717-726.

Payne, S., Sheldon, F., Jarrett, N., Large, S., Smith, P., Davis, C. L., et al. (2002). Differences in Understanding of Specialist Palliative Care Amongst Service Providers and Commissioners in South London. Palliative Medicine, 16(5), 395-402.

Pirolli, P., \& Card, S. (1995). Information Foraging in Information Access Environments. Interchi, 5158. Human Factors in Computing Systems: CHI '95 Conference Proceedings. Available from http://www.acm.org/sigchi/chi95/Electronic/documnts/papers/ppp_bdy.htm.

Pirolli, P., Card, S. K. (1998). Information Foraging Models of Browsers for Very Large Document Spaces. Paper presented at the Working Conference on Advanced Visual Interfaces, L'Aquila, Italy. Available from http://www2.parc.com/istl/projects/uir/pubs/items/UIR-1998-10-Pirolli-AVI98InformationForaging.pdf.

Raholm, M. B., Lindholm, L. Eriksson, K. (2002). Grasping the Essence of the Spiritual Dimension Reflected through the Horizon of Suffering: An Interpretative Research Synthesis. Australian Journal of Holistic Nursing, 9(1), 4-13.

Rosenfeld, K. E., Wenger, N. S., \& Kagawa-Singer, M. (2000). End-of-Life Decision Making: A Qualitative Study of Elderly Individuals. Journal of General Internal Medicine, 15(9), 620-625.

Teno, J. M., Casey, V. A., Welch, L. C., \& Edgman-Levitan, S. (2001). Patient-Focused, FamilyCentered End-of-Life Medical Care: Views of the Guidelines and Bereaved Family Members. Journal of Pain and Symptom Management, 22(3), 738-751.

Teno, J. M., Field, M. J., \& Byock, I. (2001). Preface: The Road Taken and to Be Traveled in Improving End-of-Life Care. Journal of Pain and Symptom Management, 22(3), 713-716.

Thibault, J. (2003). How Can Health Care Professionals Meet the Spiritual Needs of Dying Older Patients? Geriatric Times, I(4).

Werth, J. L., Blevins, D., Toussaint, K. L., \& Durham, M. R. (2002). The Influence of Cultural Diversity on End-of-Life Care and Decisions. American Behavioral Scientist, 46(2), 204-219.

White, A. (2002). Ethnic Group Classification. London: Her Majesty's Stationery Office (HMSO).

Wilkes, L. (1998). Palliative Care Nursing Research: Trends from 1987 to 1996. International Journal of Palliative Nursing, 4(3), 128-134.

Yin, R. K. (1989). Case Study Research: Design and Methods. Newbury Park, London: Sage Publications. 